\title{
Simplified heavy metal staining techniques demonstrated with Fast Plant leaf tissue
}

HARRIS Joseph B.. Thomas G. GUILLIAMS and JefFery

A. SCHULTZ

Biology Department, University of Wisconsin-Stevens Point, Stevens Point, WI 54481, USA.

\begin{abstract}
Fast Plant (Brassica rapa, Cruciferae) leaf tissue fixed in glutaraldehyde-acrolein and post-fixed in osmium, was examined for response to several easilyprepared heavy metal stains. Lead and uranium, separately and in combination, gave typical results across the spectrum of cell organelles. As a single stain following osmium, bismuth produced images seemingly equivalent to lead and uranium. Phosphotungstic acid produced very good membrane delineation but produced a washed-out background image similar to that from lead staining. Carbohydrate compounds were especially responsive to ruthenium; the cytoplasm and the matrix of all organelles were also stained very well. The procedures were no more demanding than traditional staining methods and may be easily used in research and teaching. Fast Plant materials are a reliable, quick and easy source of living material.
\end{abstract}

Key words: Fast Plant leaf tissue, simplified heavy metal staining technique.

\section{INTRODUCTION}

Alternative staining methods in transmission electron microscopy which provide different informetion are of wide value in both research and teaching. Methods development in electron microscopy have, in most laboratories, involved variations specifically suited for the subject. This report describes the results of using several staining methods on ultrathin sections which had been prepared by the application of traditional fixation and embedding procedures. Ease of preparation and use of stains was an objective.

Fast Plant genetic stock was orginally selected from populations of Brassica rapa (Cruciferae, mustard family). The advantage of the selection was the short 
Simplified heavy metal staining techniques

life cycle which was five weeks from seed to seed [1]. Tissue froms these plants have been shown to be used easily for ultrastructural studies with traditional methods [2]. This report describes the response of Fast Plant leaf tissues to several simple staining procedures.

It was considered important to use standard fixation procedures so as to both introduce minimum methodological encumbrance and allow for ease in comparison with familiar images.

\section{MATERIALS AND METHODS}

\section{Fixation, Embedding, Sectioning.}

Fast Plant seedlings were grown on filter paper in finger bowls to a useful size within 60 hours at room temperatures and ordinary lighting. Whole leaflets or other plant parts, were cut and dropped into vials filled to $1 /[5]$ volume with $3 \%$ glutaraldehyde and $3 \%$ acrolein in $0.02 \mathrm{M}$ sodium cacodylate bufer at $\mathrm{pH} 7.4$ and $0-3{ }^{\circ} \mathrm{C}$ (3). Specimens were submerged under a stainless steel screen and vacuum was applied and released until air was removed and infiltration complete. Fixation was continued for two hours, followed by two hours of rinsing in $0.02 \mathrm{M}$ cacodylate buffer ( $8-10$ changes), two hours postfixing in $2 \%$ osmium tetroxide in $0.02 \mathrm{M}$ cacodylate buffer, and four rinses in glass-distilled water. Dehydration in an acetone series (3 rinses each) was followed by addition of an equal volume of Spurr's resin mix (4) to the third change of $100 \%$ acetone. Specimens were mixed well, allowed to stand for 30 minutes and a second equal volume of resin was added. After thorough mixing, and standing for 30 minutes the resin was poured off', tissues were transferred to $100 \%$ resin, mixed and infiltrated overnight. Subsequently tissue strips were cut into $1 \mathrm{~mm}$ squares, transferred to plastic molds, the molds filled and labeled, and the resin polymerized for eight hours in a $70^{\circ} \mathrm{C}$ vacuum oven. Tissues were sectioned with a Sorvall MT-2B ultramicrotome and DuPont diamond knife.

\section{Staining.}

Since osmium was used as a postfixative, it characterized all images as a stain. Thus other stains were an addition to osmium. Most stains were applied by floating the grids on a drop of solution. Uranyl acetate [5] was used as an aqueous $2 \%$ solution ( $\mathrm{pH} 5)$ for 20 minutes. Reynold's formulation [6] of lead citrate(pH12), was applied for 30 minutes. A hot water rinse insured removal of carbonate crystals. Sections were immersed in the bismuth formulation [7] for 10 minutes. Treatment in 1\% phosphotungstic (PTA) acid in 95\% ethanol for five minutes followed by thorough rinsing in water gave good results. 2\% PTA in $80 \%$ or $100 \%$ ethanol has been used by other workers, and $\mathrm{pH}$ variations have given slight variations in results [8]. Staining for 60 minutes in a $0.5 \%$ solution of ruthenium red prepared in $0.1 M$ ammonium hydroxide [9] followed by a brief distilled water rinse procluced definitive images.

\section{RESULTS AND DISCUSSION}

Figures $1-4$ show the effects of traditional staining procedures using osmium, lead and uranium. Fast Plant leaf organelles were selectively responsive to these stains. Since osmium is widely believed to bind with the unsaturated double bonds of both fatty acids and other molecules, it is not surprising that there was an absence of stain in the cell wall (Fig. 1). This structure is more than $75 \%$ carbohydrate and very low in protein. The middle lamella, composed of pectin (a pentose polymer), was barely visible with osmium. Whereas the cell membrane was lightly stained, those of the vacuoles, nuclei, chloroplasts and mitochondria were significantly differentiated. The thylakoid "layers" of the chloroplasts were especially conspicuous, probably owing to their chlorophyll lipoprotein composition. Both the nucleoplasm and cytoplasm showed a very fine-grained appearance. 
Heterochromatin was hardly visible. The significant appearance of osmium in membrane patterns indicated that the leaf tissue was well preserved, allowing sufficient csmium bonding to become easily visible.

Lead staining is enhanced both by fixation with osmium and by the high $\mathrm{pH}$ of the Reynold's formulation [8]. These conditions create sites for the addition of the cation. Further, reduced osmium attracts lead to membrane sites which are thereby significantly enhanced. Since the cell wall (Fig. 2) was heavily stained with lead it is assumed to be a reaction through hydrogen bonding with the mixed sugars and pectin. This has been proposed as well for the bonding with liver glycogen. In addition to overall enhancement of membranes, the ground substance, especially of mitochondria was improved by lead. Yet cytoplasmic contents showed a washed-out appearance. The RNA-containing nucleolus displayed a clear granularity, which is typical of RNA nucleoproteins.

Uranium increased differentiation of the RNA-containing ribosomes and nucleoli. There was also enhancement of heterochromatin (Figs. 3 and 7). The middle lamella was lightly stained (Fig. 7). Otherwise uranium improved the image only slightly, over osmium. This might be expected since uranium may not bind at all with membrane or other protein. It may be used to differentiate between DNA and RNA but this requires very careful control over staining time [8].

All three commonly used stains provided the typical image of a plant leaf cell (Fig. 4). The cell wall was heavily stained with lead, membranes of all organelles with osmium and lead, and ribosomes with lead and uranium. In addition, Fig. 4 shows that starch of the chloroplasts is heavily stained. This is unusual but has been shown for young chloroplasts of different genera [10, 11]. Chloroplast thylakoids were stained heavily in contrast with the matrix which showed the washed-out appearance typical of lead treatment. Cellular cytoplasm and to a lesser extent nucleoplasm showed a leached appearance as from lead alone (Fig. 4).

Bismuth as a single stain following osmium, was equivalent in many respects to both lead and uranium (Figs. 5 and 9). It stained at least one layer in the cell wall and membranes of all organelles. Chloroplast starch and thylakoids, nucleoli and all ribosomes were superior in contrast, and membranes were equivalent in contrast to images from other stains (Fig. 9). Bismuth is known to bind both with basic amino groups, free or in proteins, and with phosphates. It's staining of ribosomes has been claimed to be blocked by glutaraldehyde fixation [12], yet Figs. 5 and 9 show distinct stain specificity. This may be due to bismuth enhancement of osmium [13].

Phosphotungstic acid (PTA), applied as 1\% in 95\% ethanol, showed generally very good, but not superior, cytoplasmic and organelle membrane staining (Fig. 6). It also heavily stained at least one fraction of the chloroplast matrix as well as heterochromatin, but a significant fraction of the background of all organelles was unstained. This produced a washed out appearance, and this may be of value in some research studies. The absence of the typical negative-stain appearance is due to osmium staining, especially of membranes. There was almost a complete absence 
Simplified heavy metal staining techniques

of stain in both starch and in the cell wall which indicated a lark of affinity by the stain for carbohydrates, either in this formulation or with the Fast Plant leaf tissue. PTA is reported to bend with alcohol, carboxyl and amino groups [8]. Alcohol concentration, $\mathrm{pH}$ and staining time are variables which should be explored on an individual tissue basis [8].

Ruthenium, which has been widely used in light microscopy for mucopolysaccharides both on the cell surface and on membranes, has also been used successfully in electron microscopy. Fig. 8 shows it to be a good nonspecific membrane and background stain with certain peculiarities. Most conspicuous are the unstained or destained particulate fractions of both mitochondria and chloroplasts. These fractions appeared as irregular in outline and were of about the same thickness as the thylakoids of chloroplasts. They were located at the sites of chloroplast puncta which stained with uranium and slightly with bismuth (Figs. 5 and 7). Puncta are of unknown chloroplast significance and are considered artifactual by some authors. Apparent destaining of the chloroplastic and mitochondrial osmiophilic globules was noticeable. A second peculiar figure resulting from ruthenium staining was granular splotches on the starch grains. These seemed to be more concentrated on the surface than in the interior. Since the starch grain should be of uniform thickness in the section, this localization may represent variations in starch chemistry from its surface in vivo. Carboxyl groups have be.en thought to be the target of the stain, but the complete absence of stain in the puncta and a few other regions is difficult to explain.

\section{ACKNOWLEDGMENT}

This report was presented in part at the CEMS/EMSA workshops on electron microscopy biologic techniques in Shanghai and Beijing, October, 1990. The senior author gratefully acknowledges the productive hospitality extended by the Second Military Medical University and the Institute of Basic Medical Sciences, their staff and workshop participants.

\section{REFERENCES}

[1] Williams, P. H. and Hill, C. B. Rapid cycling populations of Brassica. Science 1986; 232: 1385-1389.

[2] Guilliams, T. G. and Harris, J. B. Fast Plant mesophyll cell ultrastructure. Midwest Microscopy $1990 ; 20: 11$.

[ 3 ] Hall, J. L., ed. Electron Microscopy and Cytochemistry of Plant Cells and Tissues. Elsevier/North Holland Biomedical Press. Amsterdam 1978.

[4] Spurr, A. R. A low-viscosity resin embedding medium for electron microscopy. Ultrastruct Res 1969; $26: 31$.

[5] Watson, M. L. Staining of tissue sections for electron microscopy with heavy metals. J Biophys Biochem Cytol 1958; 4:475.

[6] Reynolds, E. S. The use of lead citrate at high $\mathrm{pH}$ as an electron-opaque stain in electron microscopy. J Cell Biol 1963; 17:208.

[ 7 ] Ainsworth, S. K. and Karnofsky, M. J. Alkaline bismuth reagent for high resolution ultrastructural demonstration of periodate-reactive sites J Histochem Cytochem 1972; 20:995.

[ 8] Hayat, M. A. Principles and Techniques of Electron Microscopy, Biological Applications, Volume 1. Van Nostrand Reinhold Co. New Nork 1970.

[9] Vian, B. and Reis, D. Apports de l'utilization de resines hydrosolubles a l'etude des polysaccharides dans la cellular vegetale. C R Acad Sci 1972; (Paris) 274:1663. 
[10] Casadoro, G. and Rascio, N. Chloroplast ontogenesis in Helianthus annuus L. Protoplasma 1978; $97: 165$.

[11] Miyake, H. and Maeda, E. Development of bundle sheath chloroplasts in rice seedlings. Can. J Botany 1976: 54: 556.

[12] Locke, M. and Huie, P. Bismuth staining for light and electron microscopy. Tissue Cell 1977; 9:347.

[13] Wigglesworth, V. B. The union of protein and nucleic acid in the living cell and its demonstration by osmium staining. Q J Microsc Sci 1964; 105:113.

\section{Received 18-10-1990. Revised 11-3-1991. Accepted 14-3-1991.}

Fig. 1. Osmium-stained organelles. The cell wall was not stained; all membranes were stained lightly, and the nuclear envelope and thylakoids of the chloroplast heavily. Magnification: 18,880.

Fig. 2. Osmium and lead staining. Most membrane staining was enhanced, and the cell wall as well as RNAcontaining structures were especially targeted by lead. Magnification: 19,470.

Fig. 3. Osmium and uranium staining. Membranes were stained no more intensely than with osmium alone, but ribosome and cell wall staining was significantly improved. Magnification: 18,290.

$\mathrm{CW}=$ cell wall, $\mathrm{N}=$ nucleus, $\mathrm{Cl}=$ chloroplast; $\mathrm{M}=$ mitochondrion, $\mathrm{MB}=$ microbody.

Fig. 4. Osmium, lead and uranium stains clearly delineate all membranes, cell wall, starch of the chloroplast, and RNA-containing structures. As with osmium and lead alone, background staining is reduced. Magnification: 25,280.

Fig. 5. Bismuth following osmium results in enhanced contrast of all nuclear, chloroplast and mitochondrial structures. There seems to be somewhat more retention of background structure than with lead treatment. Magnification: 30,080.

Fig. 6. Phosphotungstic acid after osmium improved the osmium staining of mitochondrial membranes and nuclear nucleic acids but also resulted in leaching of ground substance throughout the celL Carbohydrates of cell wall and chloroplast were unstained. Magnification: 26, 070.

$\mathrm{CW}=$ cell wall, $\mathrm{N}=$ nucleus, $\mathrm{Cl}=$ chloroplast, $\mathrm{M}=$ mitochondrion, $\mathrm{G}=$ Golgi body.

Fig. 7. Uranium staining after osmium at high magnification shows weak staining of the middle lamella of the cell wall and puncta of the chloroplast. Membranes of the mitochondrion and chloroplast were enhanced slightly. Magnification: 49.600

Fig. 8. Ruthenium red treatment after osmium slightly enhances staining overall. In addition it seems to be specific for components of the cell wall and starch of the chloroplast. Curiously, the chloroplast puncta and granules of mitochondria seem to be destained. In addition, osmiophilic globules of the chloroplast and mitochondria are either destained or less conspicuous. Magnification: 69,300.

Fig. 9. Bismuth staining following osmium at high magnification. Membrane detail and other cell features seem equivalent in presentation to lead and uranium. Magnification: 49,400. $\mathrm{CW}=$ cell wall, $\mathrm{N}=$ nucleus, $\mathrm{Cl}=$ chloroplast, $\mathrm{T}=$ thylakoid, $\mathrm{S}=$ starch, $\mathrm{P}=$ puncta, $\mathrm{O}=$ osmiophilic globule, $\mathrm{M}=$ mitochondrion. 
Simplified heavy metal staining techniques

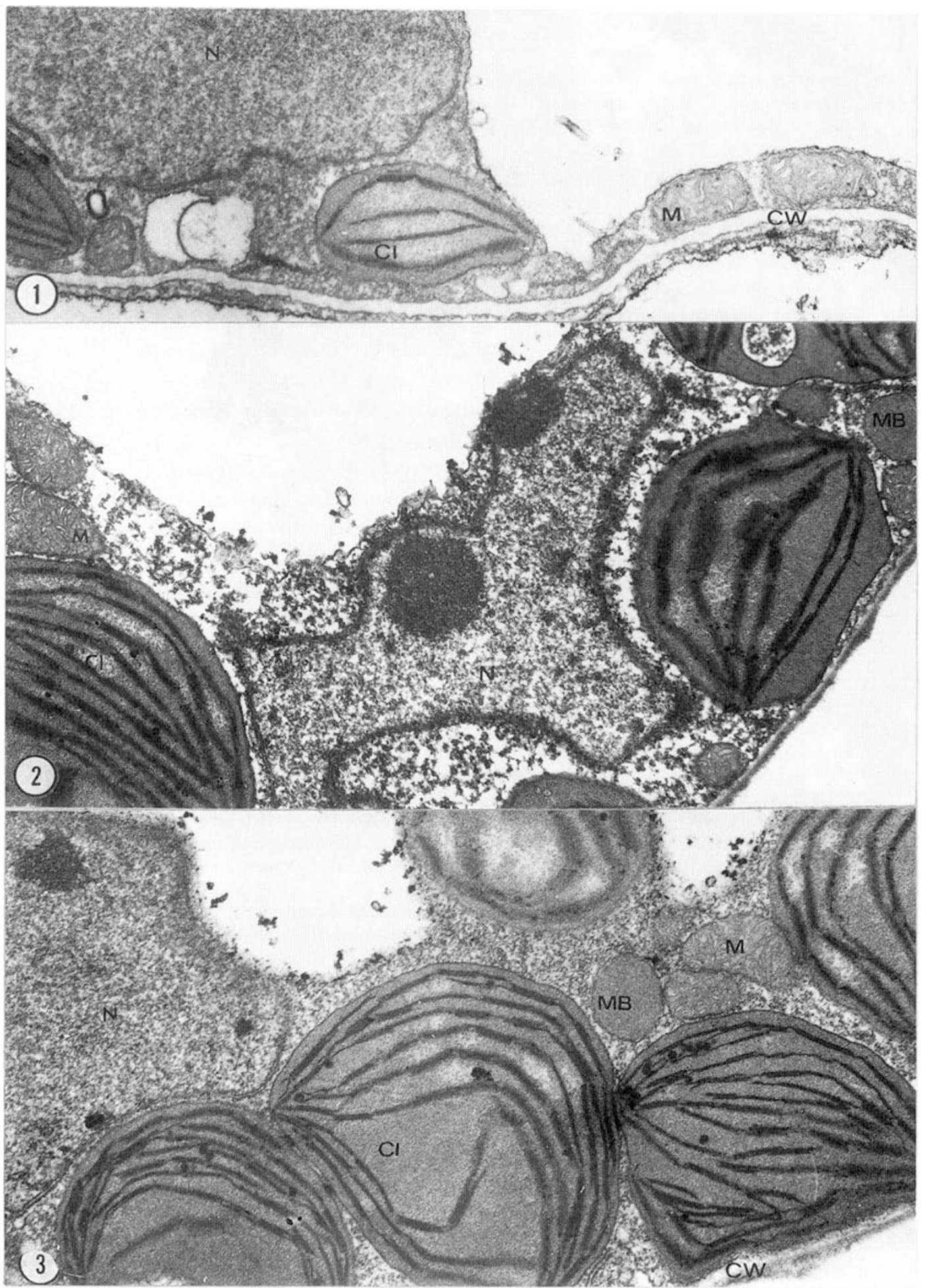


Harris JB et al.

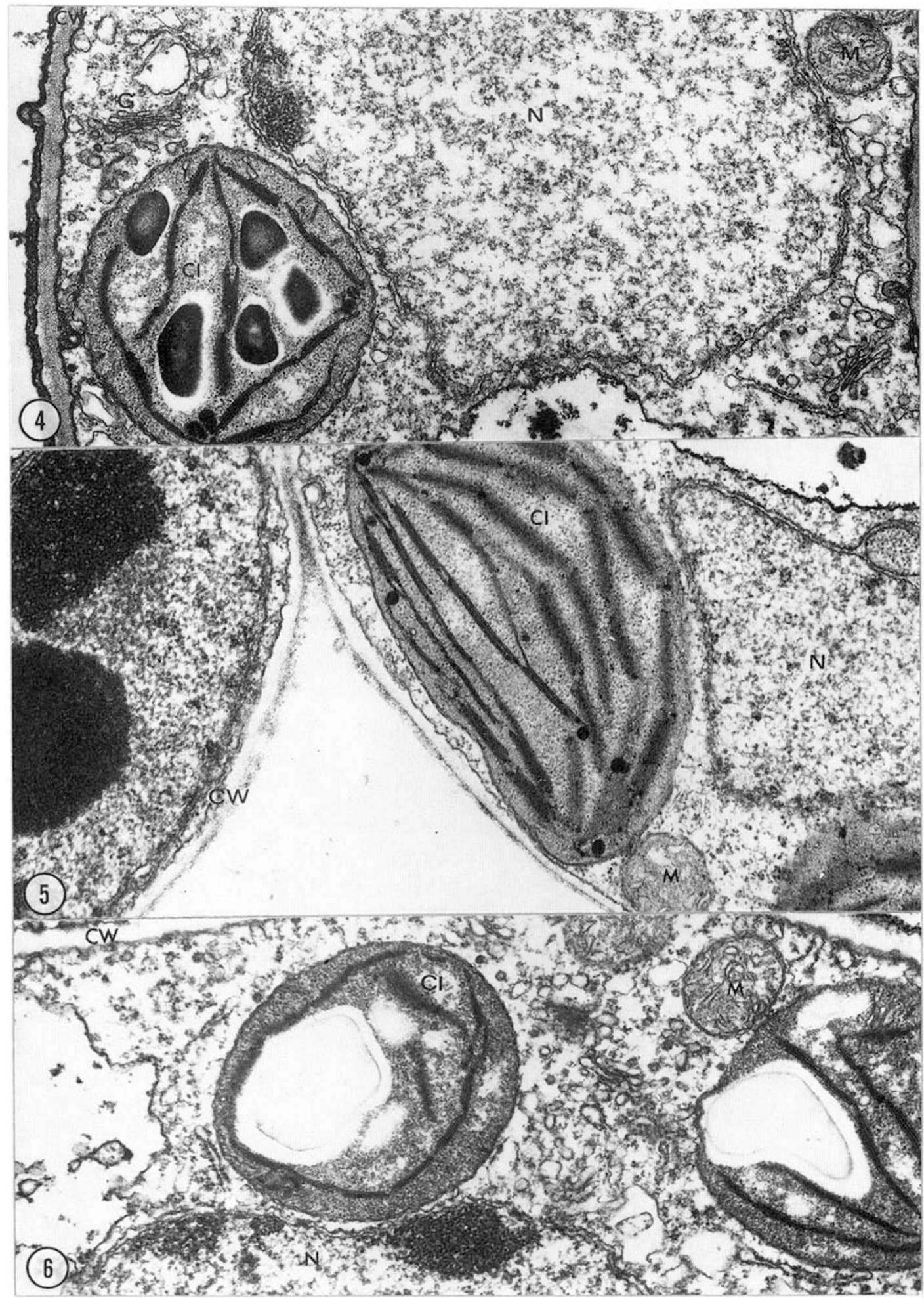


Simplified heavy metal staining techniques

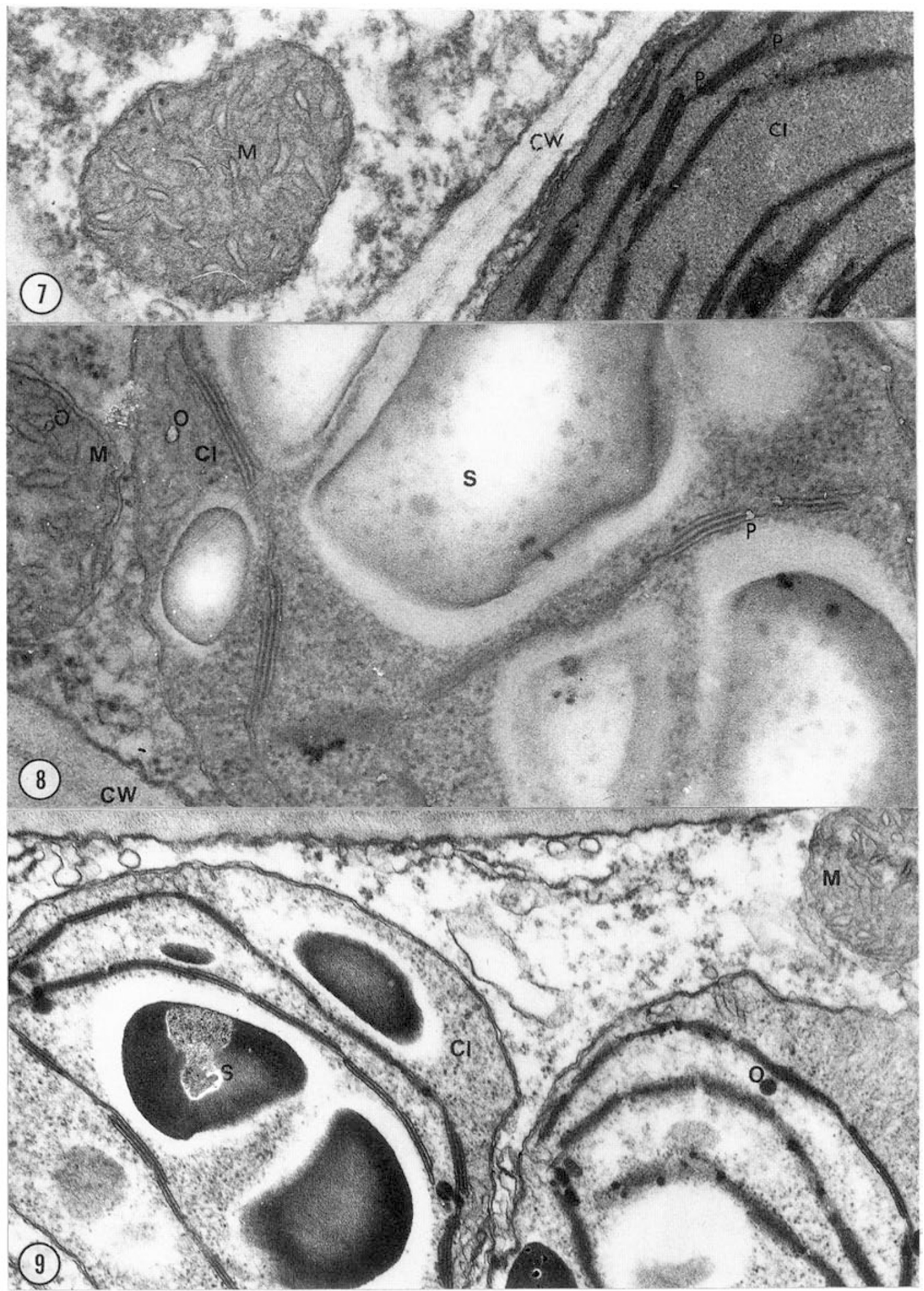

